\title{
Diet and Skin Barrier: The Role of Dietary Interventions on Skin Barrier Function
}

\author{
Milbrey A. Parke1, Ariadna Perez-Sanchez ${ }^{2}$, Dina H. Zamil ${ }^{1}$, Rajani Katta ${ }^{3}$
}

1 Baylor College of Medicine, Houston, TX, USA
2 Internal Medicine, University of Texas Health Science Center at San Antonio, TX, USA
3 Department of Dermatology, McGovern Medical School at UTHealth, Houston TX, USA

Key words: skin barrier, supplements, atopic dermatitis, diet

Citation: Parke MA, Perez-Sanchez A, Zamil DH, Katta R. Diet and skin barrier: the role of dietary interventions on skin barrier function. Dermatol Pract Concept. 2021;11(1):e2021132. DOI: https://doi.org/10.5826/dpc.1101a132

Accepted: August 16, 2020; Published: January 29, 2021

Copyright: @2021 Parke et al. This is an open-access article distributed under the terms of the Creative Commons Attribution License BYNC-4.0, which permits unrestricted noncommercial use, distribution, and reproduction in any medium, provided the original author and source are credited.

Funding: None.

Competing interests: Rajani Katta, MD, serves on an advisory board for Vichy Laboratories and is the author of a book on diet and dermatology for the general public. The other authors have no conflicts of interest to disclose.

Authorship: All authors have contributed significantly to this publication.

Corresponding author: Rajani Katta, MD, 6750 West Loop South Suite 695 Bellaire, TX 77401, USA. Email: info@kattamd.com

\begin{abstract}
Multiple research studies have examined the role of specific dietary interventions and their effects on skin barrier function. The skin barrier is one of the body's first lines of protection against environmental insults, and disruption of this natural line of defense can result in xerosis, irritation, chronic dermatitis, and other cutaneous effects. Multiple laboratory, animal, and human studies have demonstrated that certain dietary interventions have the potential to impact skin barrier function. Measurements of skin barrier function include stratum corneum hydration and transepidermal water loss. In this review, we examine this research and provide an overview of the effects of prebiotics, probiotics, fatty acids, and emerging research on other substances.
\end{abstract}

\section{Introduction}

The skin barrier is one of the body's first lines of protection against environmental insults, including allergens, chemical irritants, microbial insults, and UV radiation. Disruption of this natural line of defense can result in xerosis, irritation, chronic dermatitis, and other cutaneous effects [1]. Given the skin barrier's major role in providing protection from multiple threats, there has been much interest in strategies to improve and "strengthen" the skin barrier. A number of research studies have now examined the role of specific dietary interventions in enhancing skin barrier function. We review this research and provide an overview of the effects of prebiotics, probiotics, fatty acids, and emerging research on other substances. 


\section{The Skin Barrier}

\section{Background}

While the skin barrier has traditionally been described in anatomic terms, it can also be described in terms of its components. Anatomically, the skin barrier can be divided into 2 layers, the dermis and epidermis. Functionally, Strugar et al. have described 4 main components: the physical layer, the microbiome, the immune layer, and the chemical layer. These components are all highly dependent on each other, and proper functioning of each component of the skin barrier is crucial for its protective role [2]. Disruption may lead to or exacerbate such conditions as irritant dermatitis, atopic dermatitis $(\mathrm{AD})$, acne, and others $[3,4]$.

The physical layer of the skin is composed of the stratum corneum, which is the outermost layer of the skin. Its main purposes include protection against water loss and against the penetration of irritants [5]. This layer can be further subdivided into cells (corneocytes) and a lipid matrix. The corneocytes secrete both the lipid precursors and the enzymes to modify these precursors into ceramides, essential and nonessential fatty acids, and cholesterol [6]. These lipids fill the extracellular space by forming lamellar bilayers, thus giving the skin its water-impermeable quality [6,7]. For the physical component of the barrier to function properly, it is believed that these lipids should be in a proportion of $50 \%$ ceramides, $25 \%$ cholesterol, and $10 \%-20 \%$ free fatty acids [8]. In addition, the omega-3/ omega- 6 fatty acid ratio is important for barrier function, with a higher ratio of omega-3 being more favorable [9]. It is also believed that a decrease in stratum corneum thickness and differentiation is part of the pathogenesis of sensitive skin [10].

The chemical layer, distributed throughout the physical layer, contains natural moisturizing factors, defense chemicals, and components that contribute to the acidic $\mathrm{pH}$ of the skin. The $\mathrm{pH}$ of healthy skin is between 4-6, and is important for the function of the many microbes that live on the skin [2]. At an alkaline $\mathrm{pH}$, which may result from the use of some moisturizers, the microbes and enzymes of the skin do not function properly. An alkaline $\mathrm{pH}$ also activates $\mathrm{Th} 2$-producing endogenous serine proteases, which further disrupt the barrier [6]. Crucial to all of this is the protein filaggrin, which helps strengthen the corneocyte cell envelope, form lamellar bodies, and maintain the acidic $\mathrm{pH}$ [6].

The microbiome layer is composed of living organisms. Flora such as Staphylococcus epidermidis and Corynebacterium commonly predominate. However, disruption of the normally acidic chemical environment facilitates colonization by Staphylococcus aureus and Staphylococcus pyogenes, which may play a role in the pathogenesis of diseases such as atopic dermatitis [2,7].

The cells that make up the immune "layer" are always alert for danger signals, such as breaks in the skin barrier or signals sent by bacteria. If a threat is sensed, they can activate to repel microbes [2]. Skin barrier damage also triggers cytokine secretion, which then acts to regenerate the skin barrier by facilitating the growth and differentiation of keratinocytes [11,12].

\section{Measurement of the Skin Barrier}

Many methods of measuring skin barrier function exist. Despite the many methods available, the optimal method of measurement is not well characterized, and conflicting results have been noted when evaluating the utility and accuracy of some methods. These uncertainties must be considered when evaluating the results of any dietary interventions on skin barrier function.

The most commonly used methods measure the physical layer of the skin barrier. The parameters targeted most often are stratum corneum hydration $(\mathrm{SCH})$ and transepidermal water loss (TEWL). SCH measures the static water-holding capacity of the skin, while TEWL measures dynamic water losses [13]. Research indicates that both of these measures may be necessary to fully characterize the functioning of the skin barrier, and they are often used together. $\mathrm{SCH}$ is the amount of water contained in the stratum corneum. A few different methods are used to measure this parameter. These include electrical measurements such as capacitance, electrical impedance, and conductance [14]. Capacitance in particular measures surface hydration, but does not give information about the actual water content or thickness of the skin [5]. A newer method is Raman spectroscopy paired with multiphoton microscopy, which can measure full-thickness hydration of the stratum corneum $[5,15]$. Studies on evaluation of $\mathrm{SCH}$ reveal limitations of these measurement methods. Another measure of skin barrier function is that of transepidermal water loss (TEWL), which measures the insensible water loss through the skin and provides information on the lipid properties of the skin [14]. This method has been validated for in vivo study of skin permeability [16] although the utility of this method for in vitro testing is questionable.

\section{Synbiotics: Prebiotics with Probiotics}

Synbiotics are a combination of probiotics and prebiotics, and their benefits are theorized to be due to effects on the gut microbiome. Among other effects, beneficial gut microbes produce short chain fatty acids, which help improve epithelial barrier function and modulate the inflammatory response $[17,18]$. Phenols, in contrast, are considered markers of a disturbed gut microbiome and have been demonstrated to disrupt keratinocyte differentiation in mice [19].

Probiotics are live microorganisms that are thought to have health benefits [20]. Lactobacillus, Bifidobacterium, and 
Saccaromyces species are among the most common microbes found in probiotics, and many but not all are found in human flora [21,22]. Prebiotics such as galactooligosaccharides (GOS) are substances which, when ingested, preferentially improve the growth of beneficial gut microbes.

Clinical studies of synbiotics in the treatment of AD have been promising, but little information is available on their skin barrier effects. In a meta-analysis of 6 studies, synbiotics with mixed bacterial strains were helpful for the treatment of $\mathrm{AD}$ in adults and children over the age of 1 year [23]. In a separate study, skin barrier function was measured. In this randomized controlled trial (RCT), consumption of the prebiotic GOS along with a probiotic milk for 4 weeks led to improved skin hydration. Serum phenol levels were also restored [24].

\section{Probiotics}

The use of probiotics in treating inflammatory and infectious diseases of various organ systems has been of increasing interest, including in dermatology [25]. A potential role in the treatment of acne [26] and AD has been explored [27,28]. Their role in treatment may be in part due to their effect on the skin barrier.

The effect of probiotics on the skin barrier may be linked to their effects on immune regulation and the expression of skin barrier proteins. Studies in mice and skin models have shown decreases in pro-inflammatory cytokines, as well as improvement in skin hydration, TEWL, and the production of skin barrier proteins. In an ex vivo skin model, the topical application of both the lysate and whole preparations of a Lactobacillus species (L. reuteri DSM 17937) decreased production of IL-1 and IL-8 [25]. This also upregulated the gene for aquaporin 3, an important skin barrier protein that transports water and glycerol [25].

In a human epidermis model, the topically applied lysate of the probiotic Lactobacillus rhamnosus improved the expression of loricrin and filaggrin, both integral proteins of the skin barrier. This same strain decreased the destruction of desmosomes after treatment with the irritant sodium lauryl sulfate [29].

Despite these promising results, direct research on the effect of probiotics alone on the skin barrier function in humans is relatively lacking. In one RCT, skin hydration was increased and TEWL was decreased after 12 weeks of oral Lactobacillus plantarum HY7714[30]. Another RCT evaluated the effects of 2 months of oral Lactobacillus paracase $i$ NCC2461, and found improved skin barrier function recovery, as indicated by TEWL after tape stripping [31]. Another RCT compared the effects of Lactococcus lacti H61 consumption in milk compared to a conventional probiotic yogurt for 4 weeks. Both groups had an increase in skin hydration, and the probiotic milk group also had a significant increase in sebum content [32].

\section{Prebiotics}

Limited studies are available specifically examining the role of prebiotics. In one RCT, healthy adults consuming GOS for 12 weeks had greater improvement in TEWL and skin hydration compared to the placebo group [33]. In mice with allergen-induced dermatitis, treatment with GOS improved both clinical severity and TEWL [34].

Overall, more human studies are needed to establish a clear association between probiotic and prebiotic consumption and skin barrier function. Human studies to date are small. However, given promising results, further research is warranted.

\section{Omega-3 Fatty Acids}

Omega-3 fatty acids include alpha-linolenic acid (ALA), eicosapentaenoic acid (EPA), and docosahexaenoic acid (DHA). Derivatives of omega-3 fatty acids are thought to influence the skin barrier by acting as transcription factors and decreasing inflammation as immune modulators [35].

Studies in skin models and cultured human keratinocytes suggest that DHA can increase filaggrin expression, attenuate inflammation, and improve epidermal keratinocyte differentiation [36-38]. An animal study demonstrated decreased TEWL, increased skin hydration, and decreased skin barrier alteration by acetone [39]. EPA and the ester of EPA have been shown in animal studies to influence ceramides in skin [40,41]. However, studies have utilized different methodologies and have shown varying improvement [36-38].

Several small human trials have examined the effects of oils rich in fatty acids. Flaxseed oil, high in ALA, was shown to improve TEWL, skin hydration, skin scaling, and roughness in female study subjects after 12 weeks of daily consumption [42]. In another study, improvements in TEWL, skin hydration, and skin sensitivity were noted [9]. In both studies, the proportion of plasma ALA in those consuming flaxseed oil increased.

Another RCT evaluated hempseed oil ingestion in those with AD. Hempseed oil contains both omega- 6 and omega- 3 fatty acids, in a ratio of about $2: 1$. Subjects reported improved skin dryness and less use of dermal medications with the hempseed oil. Although there was a trend to improved TEWL, this was not significant [43].

Limited human trials have studied the effects of fish oils specifically on TEWL. One RCT found that use of DHA for 8 weeks led to significant improvement in clinical findings of $\mathrm{AD}$ and an increase in plasma levels, but did not evaluate TEWL [44]. Another RCT found that a 4-month supplementation with fish oils led to an overall $30 \%$ improvement in clinical AD scores, although this was similar to a comparison group given corn oil. Of note, both groups had a baseline of 
significantly lower levels of serum fatty acids compared to patients with psoriasis [45].

While these studies are small, they suggest that consumption of oils rich in omega- 3 fatty acids have the potential to improve skin barrier function, possibly in those with lower serum levels at baseline. Larger studies in humans are needed to assess the magnitude of effect of these treatments.

\section{Evening Primrose and Borage Oils}

Evening primrose oil (EPO) and borage oil (BO) have been studied for the treatment of $\mathrm{AD}$, with overall disappointing results [46]. However, while many AD studies have focused on measures of clinical disease severity, few have investigated their effects on skin barrier function directly. Of these, some have reported promising results.

Gamma-linolenic acid (GLA) is an omega-6 fatty acid that has exhibited beneficial properties. EPO contains $8 \%-10 \%$ GLA and BO contains up to $25 \%$ GLA. [47,48]. One study described a negative correlation between TEWL and serum levels of GLA in children with AD. The study also noted a correlation between barrier dysfunction severity and deficiencies in essential omega- 6 fatty acids such as GLA $[49,50]$.

Human skin lacks the enzyme delta 6-desaturase, which converts linoleic acid (LA) to GLA [51-53]. As such, the skin depends on GLA created in the liver [53]. Oral supplementation of EPO and BO supplies GLA directly $[47,48]$.

It is believed that the benefits of EPO and BO may be due to the influence of GLA and its metabolites on regulatory mechanisms of barrier function [53]. Metabolites of GLA exhibit anti-inflammatory properties and are linked to increased ceramide synthesis and improved skin barrier function $[47,49,54,55]$. They also inhibit the formation of leukotriene (LT) $\mathrm{B}_{4}[49,56-58]$.

Clinical studies of EPO and BO have varied widely, with differing populations and doses. However, multiple studies have demonstrated significant beneficial effects on TEWL $[42,53,55,59]$. Stratum corneum hydration, overall, has not shown significant increases $[53,55,60]$.

Negative results have been reported as well, as 2 placebocontrolled trials found no differences in skin barrier function after GLA supplementation [49,60]. One of these, however, did find significant improvement in TEWL in a subgroup. These patients at baseline had low serum levels of a GLA metabolite, suggesting the importance of baseline status in determining who might benefit from supplementation [49].

Overall, while EPO and BO supplementation has not shown benefit for the treatment of $\mathrm{AD}$, they have shown some promise in enhancing skin barrier function. Further research should examine baseline levels of GLA that may benefit from supplementation, as well as comparing dosing regimens.

\section{Emerging Interventions}

\section{L-Histidine}

Limited research has been performed on 1-histidine supplementation, but results from 1 trial are promising and further research is warranted. L-histidine is an amino acid that is rapidly incorporated into filaggrin. The pathogenesis of $\mathrm{AD}$ has been linked to deficiencies in filaggrin, with loss of function mutations in filaggrin genes showing a strong association with AD [61]. In one small RCT with 24 adult AD patients, once-daily oral 1-histidine for 4 weeks significantly increased both filaggrin formation and skin barrier function. AD severity as measured by SCORAD improved as well, with no improvement in the placebo group [62].

\section{Combination Products}

While some combination products have shown a reduction in TEWL, the limited available research, small sample sizes, and use of multi-ingredient products limit our conclusions. This includes studies on a combination turmeric and herbal product [63], a combination borage oil, fermented milk, and vitamin product [54], and a proprietary fish oil blend with vitamin D, antioxidant mixture, and other oils [64].

\section{Conclusions}

Several dietary interventions have been studied for their potential impact on the skin barrier, primarily in the form of dietary supplements. Laboratory and animal studies have described potential mechanisms of action. While some benefits have been seen in clinical studies, a number of questions remain. Clinical trials to date have utilized small sample sizes, and many have used varying dosing regimens. Some studies have evaluated multiple active ingredients, making it difficult to isolate the effects of a single ingredient. In addition, several interventions have been studied in disease states, and therefore may not be applicable to those without skin disease. Some studies have found that a baseline status of nutrients impacts clinical outcomes and is an important variable that should be studied further in future clinical trials. It is notable that few studies have investigated the role of dietary patterns on skin barrier function. This is an important area for future study. Research studies have indicated, for example, that changes in diet can impact the gut microbiome within 1 day [65], with potential implications for the skin barrier. Current research has been focused on interventions with dietary supplements and a few foods, but further research on the effects of foods and dietary patterns on the skin barrier is warranted. Although questions remain about dosing, duration, and which populations may benefit, results to date for some interventions have been promising and further research is appropriate. 


\section{References}

1. Kim BE, Leung DYM. Significance of skin barrier dysfunction in atopic dermatitis. Allergy Asthma Immunol Res. 2018;10(3):207215. DOI: 10.4168/aair.2018.10.3.207. PMID: 29676067.

2. Strugar TL, Kuo A, Seité S, Lin M, Lio P. Connecting the dots: from skin barrier dysfunction to allergic sensitization, and the role of moisturizers in repairing the skin barrier. J Drugs Dermatol. 2019;18(6):581. PMID: 31251552.

3. Lyons JJ, Milner JD, Stone KD. Atopic dermatitis in children: clinical features, pathophysiology and treatment. Immunol Allergy Clin North Am. 2015;35(1):161-183. DOI: 10.1016/j. iac.2014.09.008. PMID: 25459583.

4. Rocha MA, Bagatin E. Skin barrier and microbiome in acne. Arch Dermatol Res. 2018;310(3):181-185. DOI: 10.1007/s00403017-1795-3.

5. Ogawa-Fuse C, Morisaki N, Shima K, et al. Impact of water exposure on skin barrier permeability and ultrastructure. Contact Dermatitis. 2019;80(4):228-233. DOI: 10.1111/cod.13174.

6. Sajić D, Asiniwasis R, Skotnicki-Grant S. A look at epidermal barrier function in atopic dermatitis: physiologic lipid replacement and the role of ceramides. Skin Ther Lett. 2012;17(7):6-9. PMID: 22825649.

7. Elias PM, Wakefield JS, Man M-Q. Moisturizers versus current and next-generation barrier repair therapy for the management of atopic dermatitis. Skin Pharmacol Physiol. 2019;32(1):1-7. DOI: 10.1159/000493641. PMID: 30336483.

8. Sugarman JL. The epidermal barrier in atopic dermatitis. Semin Cutan Med Surg. 2008;27(2):108-114. DOI: 10.1016/j. sder.2008.04.005.

9. Neukam K, De Spirt S, Stahl W, et al. Supplementation of flaxseed oil diminishes skin sensitivity and improves skin barrier function and condition. Skin Pharmacol Physiol. 2011;24(2):67-74. DOI: 10.1159/000321442.

10. Raj N, Voegeli R, Rawlings AV, et al. A fundamental investigation into aspects of the physiology and biochemistry of the stratum corneum in subjects with sensitive skin. Int J Cosmet Sci. 2017;39(1):2-10. DOI: 10.1111/ics.12334.

11. Nickoloff BJ, Naidu Y. Perturbation of epidermal barrier function correlates with initiation of cytokine cascade in human skin. $J$ Am Acad Dermatol. 1994;30(4):535-546. DOI: 10.1016/s01909622(94)70059-1. PMID: 7512582.

12. Marionnet C, Bernerd F, Dumas A, et al. Modulation of gene expression induced in human epidermis by environmental stress in vivo. J Invest Dermatol. 2003;121(6):1447-1458. DOI: 10.1111/j.1523-1747.2003.12629.x. PMID: 14675196.

13. Young MM, Franken A, Plessis JL du. Transepidermal water loss, stratum corneum hydration, and skin surface $\mathrm{pH}$ of female African and Caucasian nursing students. Skin Res Technol. 2019;25(1):88-95. DOI: 10.1111/srt.12614.

14. Jansen van Rensburg S, Franken A, Du Plessis JL. Measurement of transepidermal water loss, stratum corneum hydration and skin surface $\mathrm{pH}$ in occupational settings: A review. Skin Res Technol. 2019;25(5):595-605. DOI: 10.1111/srt.12711.

15. Crowther JM, Sieg A, Blenkiron P, et al. Measuring the effects of topical moisturizers on changes in stratum corneum thickness, water gradients and hydration in vivo. Br J Dermatol. 2008;159(3):567-577. DOI: 10.1111/j.1365-2133.2008.08703.x.

16. Fluhr JW, Feingold KR, Elias PM. Transepidermal water loss reflects permeability barrier status: validation in human and rodent in vivo and ex vivo models. Exp Dermatol. 2006;15(7):483-492. DOI: 10.1111/j.1600-0625.2006.00437.x.

17. Ellis SR, Nguyen M, Vaughn AR, et al. The skin and gut microbiome and its role in common dermatologic conditions. Microorganisms. 2019;7(11):550. DOI: 10.3390/microorganisms7110550. PMID: 31717915.

18. Zheng L, Kelly CJ, Battista KD, et al. Microbial-derived butyrate promotes epithelial barrier function through IL-10 receptor-dependent repression of claudin-2. J Immunol Baltim Md 1950. 2017;199(8):2976-2984. DOI: 10.4049/jimmunol.1700105. PMID: 28893958.

19. Miyazaki K, Masuoka N, Kano M, Lizuka R. Bifidobacterium fermented milk and galacto-oligosaccharides lead to improved skin health by decreasing phenols production by gut microbiota. Benef Microbes. 2014;5(2):121-128. DOI: 10.3920/BM2012.0066. PMID: 23685373.

20. Jeong JH, Lee CY, Chung DK. Probiotic lactic acid bacteria and skin health. Crit Rev Food Sci Nutr. 2016;56(14):2331-2337. DOI: 10.1080/10408398.2013.834874.

21. Islam SU. Clinical uses of probiotics. Medicine (Baltimore). 2016;95(5):e2658. DOI: 10.1097/MD.0000000000002658. PMID: 26844491.

22. Senok AC, Ismaeel AY, Botta GA. Probiotics: facts and myths. Clin Microbiol Infect Off Publ Eur Soc Clin Microbiol Infect Dis. 2005;11(12):958-966. DOI: 10.1111/j.1469-0691.2005.01228.x. PMID: 16307549 .

23. Chang Y-S, Trivedi MK, Jha A, Lin Y-F, Dimaano L, García-Romero MT. Synbiotics for prevention and treatment of atopic dermatitis: a meta-analysis of randomized clinical trials. JAMA Pediatr. 2016;170(3):236-242. DOI: 10.1001/jamapediatrics.2015.3943. PMID: 26810481.

24. Mori N, Kano M, Masuoka N, et al. Effect of probiotic and prebiotic fermented milk on skin and intestinal conditions in healthy young female students. Biosci Microbiota Food Health. 2016;35(3):105-112. DOI: 10.12938/bmfh.2015-022. PMID: 27508111.

25. Khmaladze I, Butler É, Fabre S, Gillbro JM. Lactobacillus reuteri DSM 17938-A comparative study on the effect of probiotics and lysates on human skin. Exp Dermatol. 2019;28(7):822-828. DOI: 10.1111/exd.13950.

26. Mottin VHM, Suyenaga ES. An approach on the potential use of probiotics in the treatment of skin conditions: acne and atopic dermatitis. Int J Dermatol. 2018;57(12):1425-1432. DOI: 10.1111/ijd.13972.

27. Lu C-L, Liu X-H, Stub T, et al. Complementary and alternative medicine for treatment of atopic eczema in children under 14 years old: a systematic review and meta-analysis of randomized controlled trials. BMC Complement Altern Med. 2018;18(1):260. DOI: 10.1186/s12906-018-2306-6. PMID: 30257693.

28. Huang R, Ning H, Shen M, Li J, Zhang J, Chen X. Probiotics for the treatment of atopic dermatitis in children: a systematic review and meta-analysis of randomized controlled trials. Front Cell Infect Microbiol. 2017;7:392. DOI: 10.3389/fcimb.2017.00392. PMID: 28932705.

29. Jung Y-O, Jeong H, Cho Y, et al. Lysates of a probiotic, Lactobacillus rhamnosus, can improve skin barrier function in a reconstructed human epidermis model. Int J Mol Sci.2019;20(17):4289. DOI: 10.3390/ijms20174289. PMID: 31480681.

30. Lee DE, Huh C-S, Ra J, et al. Clinical evidence of effects of Lactobacillus plantarum hy7714 on skin aging: a randomized, 
double blind, placebo-controlled study. J Microbiol Biotechnol. 2015;25(12):2160-2168. DOI: 10.4014/jmb.1509.09021. PMID: 26428734.

31. Gueniche A, Philippe D, Bastien P, et al. Randomised double-blind placebo-controlled study of the effect of Lactobacillus paracasei NCC 2461 on skin reactivity. Benef Microbes. 2014;5(2):137145. DOI: 10.3920/BM2013.0001. PMID: 24322879.

32. Kimoto-Nira H, Nagakura Y, Kodama C, et al. Effects of ingesting milk fermented by Lactococcus lactis H61 on skin health in young women: a randomized double-blind study. J Dairy Sci. 2014;97(9):5898-5903. DOI: 10.3168/jds.2014-7980. PMID: 25022690.

33. Hong YH, Chang UJ, Kim YS, Jung EY, Suh HJ. Dietary galacto-oligosaccharides improve skin health: a randomized double blind clinical trial. Asia Pac J Clin Nutr. 2017;26(4):613-618. DOI: 10.6133/apjen.052016.05. PMID: 28582809.

34. Weise C, Ernst D, Tol EAF van, Worm M. Dietary polyunsaturated fatty acids and non-digestible oligosaccharides reduce dermatitis in mice. Pediatr Allergy Immunol. 2013;24(4):361-367. DOI: 10.1111/pai.12073.

35. McCusker MM, Grant-Kels JM. Healing fats of the skin: the structural and immunologic roles of the $\omega-6$ and $\omega-3$ fatty acids. Clin Dermatol. 2010;28(4):440-451. DOI: 10.1016/j.clindermatol.2010.03.020.

36. Jia T, Qiao W, Yao Q, Wu W, Kaku K. Treatment with docosahexaenoic acid improves epidermal keratinocyte differentiation and ameliorates inflammation in human keratinocytes and reconstructed human epidermis models. Molecules. 2019;24(17):3156. DOI: 10.3390/molecules24173156. PMID: 31480216.

37. Wallmeyer L, Lehnen D, Eger N, et al. Stimulation of PPARX normalizes the skin lipid ratio and improves the skin barrier of normal and filaggrin deficient reconstructed skin. J Dermatol Sci. 2015;80(2):102-110. DOI: 10.1016/j.jdermsci.2015.09.012.

38. Zhang C, Zhang C, Gurevich I, Aneskievich BJ. Organotypic modeling of human keratinocyte response to peroxisome proliferators. Cells Tissues Organs. 2012;196(5):431-441. DOI: 10.1159/000336268. PMID: 22677707.

39. Barcelos RCS, de Mello-Sampayo C, Antoniazzi CTD, et al. Oral supplementation with fish oil reduces dryness and pruritus in the acetone-induced dry skin rat model. J Dermatol Sci. 2015;79(3):298-304. DOI: 10.1016/j.jdermsci.2015.06.015.

40. Kendall AC, Kiezel-Tsugunova M, Brownbridge LC, Harwood JL, Nicolaou A. Lipid functions in skin: differential effects of n-3 polyunsaturated fatty acids on cutaneous ceramides, in a human skin organ culture model. Biochim Biophys Acta. 2017;1859(9Part B):1679-1689. DOI: 10.1016/j.bbamem.2017.03.016. PMID: 28341437.

41. Fujii M, Ohyanagi C, Kawaguchi N, et al. Eicosapentaenoic acid ethyl ester ameliorates atopic dermatitis-like symptoms in special diet-fed hairless mice, partly by restoring covalently bound ceramides in the stratum corneum. Exp Dermatol. 2018;27(8):837840. DOI: $10.1111 /$ exd.13507.

42. De Spirt S, Stahl W, Tronnier H, et al. Intervention with flaxseed and borage oil supplements modulates skin condition in women. Br J Nutr Camb. 2009;101(3):440-445. DOI: http://dx.doi. org/10.1017/S0007114508020321.

43. Callaway J, Schwab U, Harvima I, et al. Efficacy of dietary hempseed oil in patients with atopic dermatitis. J Dermatol Treat. 2005;16(2):87-94. DOI: 10.1080/09546630510035832.
44. Koch C, Dölle S, Metzger M, et al. Docosahexaenoic acid (DHA) supplementation in atopic eczema: a randomized, double-blind, controlled trial. Br J Dermatol. 2008;158(4):786-792. DOI: 10.1111/j.1365-2133.2007.08430.x. PMID: 18241260.

45. Søyland E, Funk J, Rajka G, et al. Dietary supplementation with very long-chain n-3 fatty acids in patients with atopic dermatitis. A double-blind, multicentre study. Br J Dermatol. 1994;130(6):757-764. DOI: 10.1111/j.1365-2133.1994. tb03414.x. PMID: 8011502.

46. Bamford JTM, Ray S, Musekiwa A, van Gool C, Humphreys R, Ernst E. Oral evening primrose oil and borage oil for eczema. Cochrane Database Syst Rev. 2013;(4):CD004416. DOI: 10.1002/14651858.CD004416.pub2. PMID: 23633319.

47. Chung S, Kong S, Seong K, Cho Y. Gamma-linolenic acid in borage oil reverses epidermal hyperproliferation in guinea pigs. $J$ Nutr. 2002;132(10):3090-3097. DOI: 10.1093/jn/131.10.3090. PMID: 12368400.

48. Lawson LD, Hughes BG. Triacylglycerol structure of plant and fungal oils containing $\psi$-linolenic acid. Lipids. 1988;23(4):313317. DOI: 10.1007/BF02537340. PMID: 27520008.

49. Kawamura A, Ooyama K, Kojima K, et al. Dietary supplementation of gamma-linolenic acid improves skin parameters in subjects with dry skin and mild atopic dermatitis. J Oleo Sci. 2011;60(12):597-607. DOI: 10.5650/jos.60.597. PMID: 22123240.

50. Yen C-H, Dai Y-S, Yang Y-H, Wang L-C, Lee J-H, Chiang B-L. Linoleic acid metabolite levels and transepidermal water loss in children with atopic dermatitis. Ann Allergy Asthma Immunol Off Publ Am Coll Allergy Asthma Immunol. 2008;100(1):66-73. DOI: 10.1016/S1081-1206(10)60407-3. PMID: 18254485.

51. Chapkin RS, Ziboh VA. Inability of skin enzyme preparations to biosynthesize arachidonic acid from linoleic acid. Biochem Biophys Res Commun. 1984;124(3):784-792. DOI: 10.1016/0006-291x(84)91026-x. PMID: 6439197.

52. Chapkin RS, Ziboh VA, Marcelo CL, Voorhees JJ. Metabolism of essential fatty acids by human epidermal enzyme preparations: evidence of chain elongation. J Lipid Res. 1986;27(9):945-954. PMID: 3097227.

53. Brosche T, Platt D. Effect of borage oil consumption on fatty acid metabolism, transepidermal water loss and skin parameters in elderly people. Arch Gerontol Geriatr. 2000;30(2):139-150. DOI: 10.1016/S0167-4943(00)00046-7.

54. Puch F, Samson邓Villeger S, Guyonnet D, Blachon J-L, Rawlings $\mathrm{AV}$, Lassel T. Consumption of functional fermented milk containing borage oil, green tea and vitamin E enhances skin barrier function. Exp Dermatol. 2008;17(8):668-674. DOI: 10.1111/j.16000625.2007.00688.x.

55. Park KY, Ko EJ, Kim IS, et al. The effect of evening primrose oil for the prevention of xerotic cheilitis in acne patients being treated with isotretinoin: a pilot study. Ann Dermatol. 2014;26(6):706712. DOI: 10.5021/ad.2014.26.6.706. PMID: 25473222.

56. Bauer FW, van de Kerkhof PC, Maassen-de Grood RM. Epidermal hyperproliferation following the induction of microabscesses by leukotriene B4. Br J Dermatol. 1986;114(4):409-412. DOI: 10.1111/j.1365-2133.1986.tb02843.x. PMID: 3008801.

57. Iversen L, Fogh K, Bojesen G, Kragballe K. Linoleic acid and dihomogammalinolenic acid inhibit leukotriene B4 formation and stimulate the formation of their 15-lipoxygenase products by human neutrophils in vitro. Evidence of formation of antiin- 
flammatory compounds. Agents Actions. 1991;33(3-4):286-291. DOI: 10.1007/bf01986575. PMID: 1659156.

58. Chilton-Lopez null, Surette ME, Swan DD, Fonteh AN, Johnson MM, Chilton FH. Metabolism of gammalinolenic acid in human neutrophils. J Immunol Baltim Md 1950. 1996;156(8):29412947. PMID: 8609415.

59. Muggli R. Systemic evening primrose oil improves the biophysical skin parameters of healthy adults. Int J Cosmet Sci. 2005;27(4):243-249. DOI: 10.1111/j.1467-2494.2005.00274.x. PMID: 18492193.

60. Chung BY, Park SY, Jung MJ, Kim HO, Park CW. Effect of evening primrose oil on korean patients with mild atopic dermatitis: a randomized, double-blinded, placebo-controlled clinical study. Ann Dermatol. 2018;30(4):409-416. DOI: 10.5021/ad.2018.30.4.409. PMID: 30065580.

61. Irvine AD, McLean WHI, Leung DYM. Filaggrin mutations associated with skin and allergic diseases. N Engl J Med. 2011;365(14):1315-1327. DOI: 10.1056/NEJMra1011040. PMID: 21991953.
62. Tan SP, Brown SB, Griffiths CE, Weller RB, Gibbs NK. Feeding filaggrin: effects of 1-histidine supplementation in atopic dermatitis. Clin Cosmet Investig Dermatol. 2017;10:403-411. DOI: 10.2147/CCID.S146760. PMID: 29042806.

63. Vaughn AR, Clark AK, Notay M, Sivamani RK. Randomized controlled pilot study of dietary supplementation with turmeric or herbal combination tablets on skin barrier function in healthy subjects. J Med Food. 2018;21(12):1260-1265. DOI: 10.1089/ jmf.2018.0015. PMID: 30457892.

64. Segger D, Matthies A, Saldeen T. Supplementation with Eskimo Skin Care improves skin elasticity in women. A pilot study. J Dermatol Treat. 2008;19(5):279-283. DOI: 10.1080/09546630801958238. PMID: 19160533.

65. Wu GD, Chen J, Hoffmann C, et al. Linking long-term dietary patterns with gut microbial enterotypes. Science. 2011;334(6052):105-108. DOI: 10.1126/science.1208344. 
